> Les propriétés mécaniques des cellules jouent un rôle prépondérant dans de nombreux événements de la vie cellulaire comme le développement embryonnaire, la formation des tissus ou encore le développement des métastases. La migration cellulaire est en partie caractérisée par des interactions mécaniques. Ainsi, les forces de traction qu'exercent les cellules sur leur environnement impliquent, en parallèle, une réorganisation dynamique des processus d'adhérence et du cytosquelette interne de la cellule. Pour évaluer ces forces, un substrat a été développé, constitué d'un réseau forte densité de micro-piliers déformables sur lequel se déplacent les cellules. Cette surface est fabriquée par des méthodes de lithographie empruntées à la micro-électronique. Les piliers mesurent environ un micromètre et sont en caoutchouc, donc suffisamment déformables pour fléchir sous l'effet des forces exercées par les cellules. L'analyse au microscope des déflexions individuelles de chaque pilier a permis de quantifier en temps réel les forces locales que des cellules exercent sur leur substrat lors de leurs processus d'adhérence et de dissociation. <

\section{Un substrat \\ de micropiliers \\ pour étudier \\ la migration \\ cellulaire}

Axel Buguin, Philippe Chavrier, Benoit Ladoux, Olivia du Roure, Alexandre Saez, Pascal Silberzan

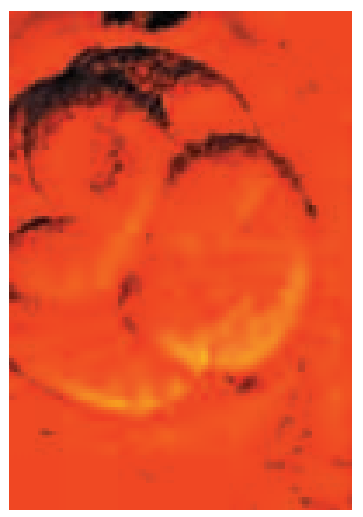

A. Buguin, P. Silberzan :

Laboratoire de Physico-chimie, CNRS UMR 168, Institut Curie, 26, rue d'Ulm, 75005 Paris, France.

P. Chavrier: Laboratoire de Compartimentation et dynamique cellulaires, CNRS UMR 144IC, Institut Curie, 26, rue d'UIm, 75005 Paris, France.

cellulaire). À l'intérieur B. Ladoux, 0. du Roure, A. Saez: du corps cellulaire, ces Matière et Systèmes Complexes, efforts sont principale- CNRS UMR 7057, Université Paris ment supportés par le 7, Case 7056, Tour 33, 2, place cytosquelette, réseau Jussieu, 75251 Paris Cedex 05, enchevêtré de plusieurs France. types de polymères ladoux@ccr.jussieu.fr (filaments d'actine, microtubules, filaments intermédiaires), véritable architecture dynamique de la cellule, qui lui confère l'essentiel de ses propriétés mécaniques. En outre, ce réseau est labile : il se remodèle en permanence par polymérisation et dépolymérisation, ou par action des moteurs moléculaires, petites protéines capables de se déplacer le long des filaments et de les faire glisser les uns par rapport aux autres. Ces mécanismes permettent de modifier la morphologie de la cellule, par exemple lorsque celle-ci se déplace.

\section{Un tapis de fakir pour sentir la motilité des cellules}

Dans ce contexte, nous nous sommes plus particulièrement intéressés au mouvement des cellules épithéliales. À terme, ces études peuvent permettre d'ouvrir de nouvelles pistes sur les processus impliqués dans la migration des cellules cancéreuses, qui envahissent les tissus en échappant aux mécanismes de régulation chargés du maintien de la posi-

Article reçu le 18 mai 2005, accepté le 30 mai 2005. 
tion des cellules dans l'organisme. Dans l'épithélium, les cellules adhèrent fortement au substrat et sont immobiles. Cependant, dans certaines situations physiologiques (développement embryonnaire, renouvellement normal du tissu épithélial, ou réparation de lésions) ou pathologiques (transformation tumorale et développement des métastases), ces cellules sont capables de se détacher les unes des autres et d'adopter un phénotype motile. Cette transformation, qui s'accompagne d'une modification des conditions dynamiques d'adhérence des cellules entre elles et avec leur support, peut être induite par l'introduction d'un facteur dispersant dans le milieu cellulaire [2].

Pour mesurer les forces exercées sur le substrat lors de la migration cellulaire, la plupart des travaux actuels utilisent des surfaces continues et déformables (gels) dans lesquelles sont insérés des marqueurs (billes fluorescentes, surfaces microfabriquées) [3]. Connaissant l'élasticité du gel, la mesure du déplacement de ces marqueurs permet de remonter aux forces. Cette approche a conduit à de nombreuses avancées dans la compréhension des mécanismes impliqués dans la migration cellulaire.
Cependant, des difficultés, dues en particulier au caractère continu de ces surfaces, empêchent d'atteindre la précision voulue sur la détermination des forces. Nous avons donc mis au point un substrat original composé d'un réseau discret de capteurs de force intégré à la surface [4]. Celle-ci se présente donc comme un assemblage très dense de petits piliers cylindriques souples, dont le diamètre mesure environ $1 \mu \mathrm{m}$, se déformant indépendamment les uns des autres sous l'effet des forces de traction développées par la cellule au cours de son mouvement (on peut se représenter cette surface sous la forme d'un «tapis de fakir ») (Figure 1). Ces plots, en élastomère silicone [5], sont fabriqués par moulage d'un substrat de silicium dans lequel les trous complémentaires ont été usinés par les techniques de microfabrication issues de la micro-électronique. La force exercée sur un plot est directement proportionnelle à sa déflexion.

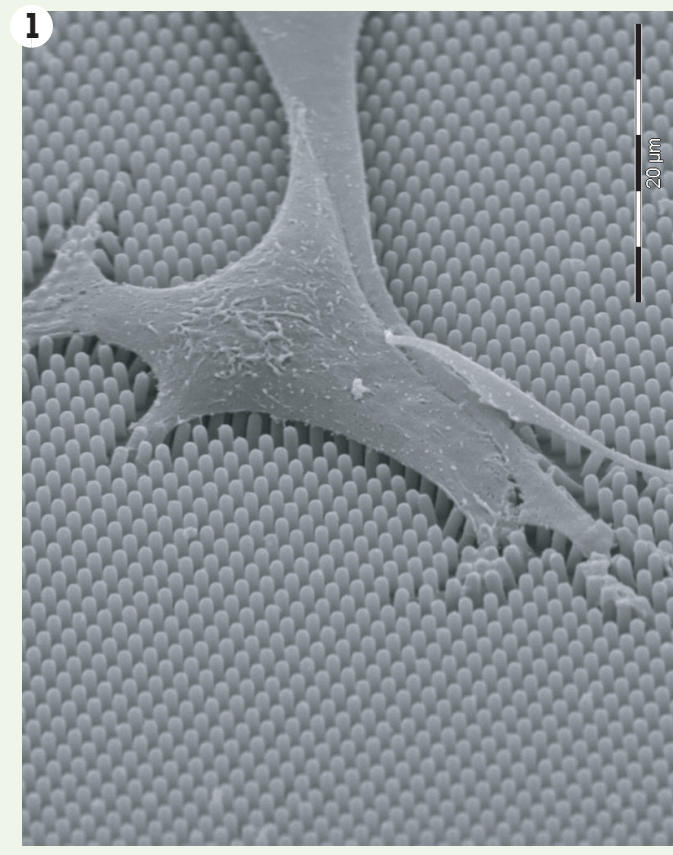

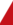

Figure 1. Cellule sur un substrat de microplots. La Iongueur de la barre d'échelle est de $20 \mu \mathrm{m}$ (microscopie électronique à balayage).

Figure 2. Trois images en microscopie par transmission d'un îlot cellulaire en croissance. L'intervalle de temps entre chaque image est de 30 minutes. Les flèches blanches représentées sur certains piliers sont proportionnelles à la déflexion et donc à la force locale exercée. La longueur de la barre d'échelle est de $10 \mu \mathrm{m}$.

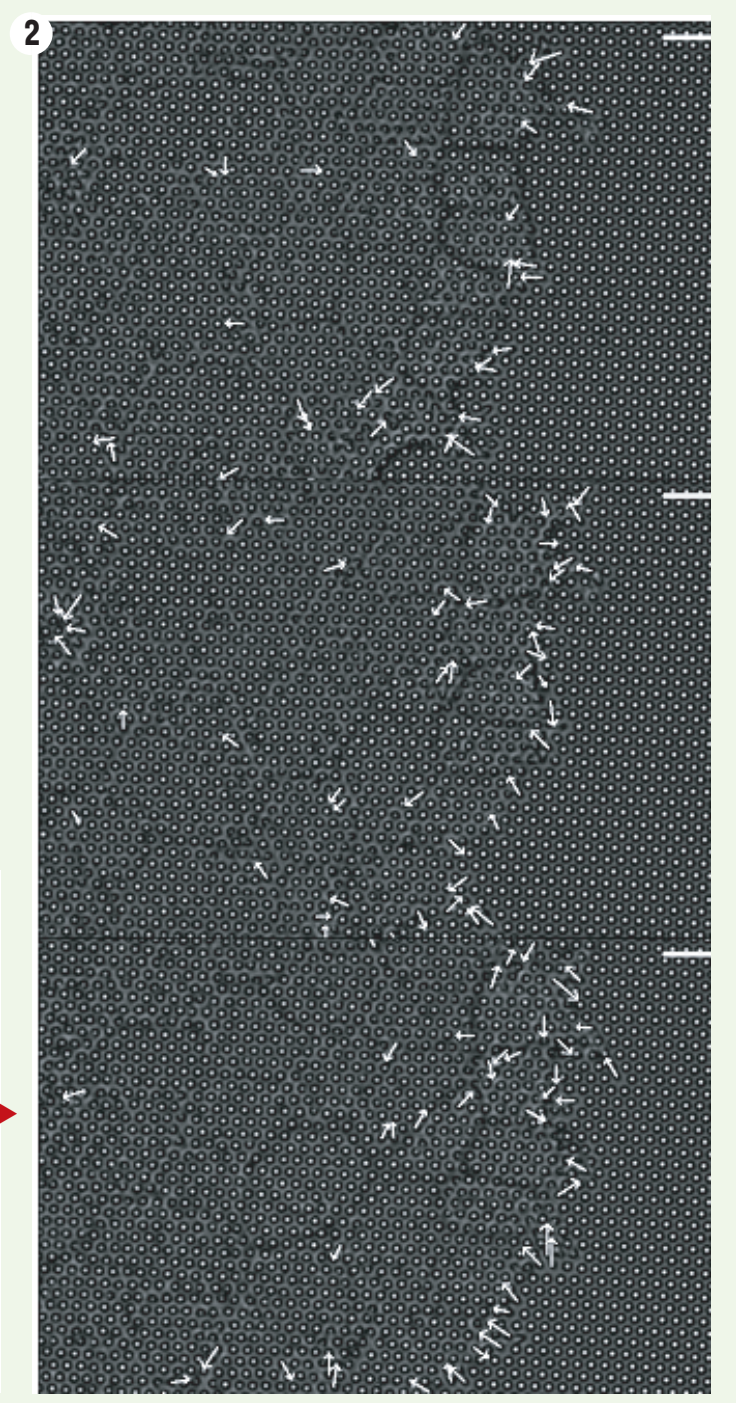


La constante de proportionnalité (la «raideur» des plots) est choisie de manière à s'adapter aux forces mises en jeu, qui sont de l'ordre de $1 \mathrm{nN}$ [6]. Avec des plots de $1 \mu \mathrm{m}$ de diamètre, une hauteur de $5 \mu \mathrm{m}$ conduit à des raideurs de l'ordre du mN/m, correspondant à des déflexions de l'ordre du micromètre, facilement observables en microscopie optique [7].

Outre sa grande flexibilité et sa biocompatibilité, l'élastomère utilisé offre l'avantage de pouvoir être traité en surface. Ces surfaces microfabriquées peuvent donc être recouvertes de protéines de la matrice extracellulaire afin de mimer le milieu naturel d'adhérence des cellules. Les cellules sont mises en culture sur ces substrats et la déflexion de chaque plot est suivie en temps réel par la vidéomicroscopie optique couplée à un traitement informatique (Figure 2): des mesures de déflexion individuelles, on remonte au champ de force.

\section{Aperçu des champs d'application}

Les travaux effectués sur les cellules épithéliales nous ont permis de caractériser le champ de forces au sein d'un îlot de quelques dizaines de cellules épithéliales. Nos mesures indiquent que les forces les plus importantes (de l'ordre de quelques dizaines de $\mathrm{nN}$ ) sont localisées sur les contours de l'îlot, la contrainte moyenne exercée par les cellules sur la surface diminuant à mesure que l'on se rapproche du centre de l'épithélium [7]. II subsiste néanmoins une activité mécanique résiduelle au sein de la monocouche, cette activité se situant essentiellement au niveau des jonctions intercellulaires.

Enfin, l'ajout de facteurs dispersants dans le milieu permet de dissocier les jonctions intercellulaires pour obtenir des cellules migrantes isolées les unes des autres. Ainsi, il nous a été possible de comparer les contraintes maximales, qui correspondent à la force exercée sur la surface disponible, développées par un ensemble de cellules épithéliales $\left(\sim 12,7 \pm 0,3 \mathrm{nN} / \mu \mathrm{m}^{2}\right)$ et une cellule isolée après dissociation $\left(\sim 3,8 \pm 0,1 \mathrm{nN} / \mu \mathrm{m}^{2}\right)$. Nos mesures montrent que les contraintes sont beaucoup plus importantes dans le cas d'un ensemble de cellules : ce renforcement peut sans doute être attribué à l'effet d'un comportement collectif.

Le développement de ces surfaces microtexturées apparaît comme un outil polyvalent pour étudier la migration cellulaire, dans la mesure où il permet de contrôler aisément les propriétés mécaniques du substrat ainsi que les propriétés chimiques de la surface. Il est maintenant important de pouvoir corréler les forces développées à des mesures d'activité des protéines intervenant dans la formation ou la dissociation des jonctions cellulaires, et donc de coupler ces mesures mécaniques à des marquages fluorescents [8]. De plus, il semble désormais acquis que la rigidité du substrat influence les propriétés d'adhérence des cellules [9]. Notre technique devrait nous permettre de caractériser les mécanismes au cours desquels la cellule sonde son environnement extérieur.

De manière plus générale, l'utilisation des techniques de microfabrication permet de tester un certain nombre d'idées et de concepts originaux sur des systèmes vivants. De ce point de vue, le développement de cette stratégie devrait rapidement apporter d'autres résultats originaux dans bon nombre de domaines de la biologie cellulaire. $\diamond$

\section{SUMMARY}

An array of microfabricated pillars to study cell migration

Mechanical forces play an important role in various cellular functions, such as tumor metastasis, embryonic development or tissue formation. Cell migration involves dynamics of adhesive processes and cytoskeleton remodelling, leading to traction forces between the cells and their surrounding extracellular medium. To study these mechanical forces, a number of methods have been developed to calculate tractions at the interface between the cell and the substrate by tracking the displacements of beads or microfabricated markers embedded in continuous deformable gels. These studies have provided the first reliable estimation of the traction forces under individual migrating cells. We have developed a new force sensor made of a dense array of soft micron-size pillars microfabricated using microelectronics techniques. This approach uses elastomeric substrates that are micropatterned by using a combination of hard and soft lithography. Traction forces are determined in real time by analyzing the deflections of each micropillar with an optical microscope. Indeed, the deflection is directly proportional to the force in the linear regime of small deformations. Epithelial cells are cultured on our substrates coated with extracellular matrix protein. First, we have characterized temporal and spatial distributions of traction forces of a cellular assembly. Forces are found to depend on their relative position in the monolayer: the strongest deformations are always localized at the edge of the islands of cells in the active areas of cell protrusions. Consequently, these forces are quantified and correlated with the adhesion/scattering processes of the cells. $\diamond$

\section{RÉFÉRENCES}

1. Lauffenburger DA, Horwitz AF. Cell migration : a physically integrated molecular process. Cell $1996 ; 84$ : 359-69.

2. Balkovetz DF. Evidence that hepatocyte growth factor abrogates contact inhibition of mitosis in Madin-Darby canine kidney cell monolayers. Life Sci $1999 ; 64: 1393-401$.

3. Beningo KA, Wang $Y \mathrm{~L}$. Flexible substrata for the detection of cellular traction forces. Trends Cell Biol $2002 ; 12: 79-84$.

4. Du Roure 0 , Dequidt C, Richert A, et al. Microfabricated arrays of elastomeric posts to study cellular mechanics. Proc SPIE Int Soc Opt Eng 2004; 5345: 26-34.

5. Tan JL, Tien J, Pirone DM, et al. Cells lying on a bed of microneedles: an approach to isolate mechanical forces. Proc Natl Acad Sci USA 2003; 100 : 1484-9.

6. Choquet D, Felsenfeld DP, Sheetz MP. Extracellular matrix rigidity causes strengthening of integrin-cytoskeletal linkages. Cell $1997 ; 88: 39-48$.

7. Du Roure 0, Saez A, Austin RH, et al. Force mapping in epithelial cell migration. Proc Natl Acad Sci USA 2005 ; 102 : 2390-5.

8. Adams CL, Chen YL, Smith SJ, et al. Mechanisms of epithelial cell-cell adhesion and cell compaction revealed by high-resolution tracking of $\varepsilon$ cadherin-green fluorescent protein. J Cell Biol 1998; 142 : 1105-19.

9. Yeug T, Georges PC, Flanagan LA, et al. Effects of substrate stiffness on cell morphology, cytoskeletal structure and adhesion. Cell Motil Cytoskeleton $2005 ; 60: 24-34$.

\section{TIRÉS À PART}

B. Ladoux 